\title{
Posttraumatic Stress Symptoms Among Chinese College Students During the COVID-19 Pandemic: A Longitudinal Study
}

\author{
Xinli Chi ${ }^{1+}$, Liuyue Huang ${ }^{1 \dagger}$, Daniel L. Hall ${ }^{2,3}$, Raissa Li ${ }^{2}$, Kaixin Liang ${ }^{1}$, \\ Md Mahbub Hossain ${ }^{4}$ and Tianyou Guo ${ }^{1 *}$ \\ ${ }^{1}$ School of Psychology, Shenzhen University, Shenzhen, China, ${ }^{2}$ Health Policy Research Center, Mongan Institute, \\ Massachusetts General Hospital, Boston, MA, United States, ${ }^{3}$ Department of Psychiatry, Harvard Medical School, Boston, \\ MA, United States, ${ }^{4}$ Department of Health Promotion and Community Health Sciences, School of Public Health, Texas A\&M \\ University, College Station, TX, United States
}

OPEN ACCESS

Edited by:

Haibo Yang,

Tianjin Normal University, China

Reviewed by:

Aiyi Liu,

Beijing Normal University, China

Arghya Pal,

All India Institute of Medical Sciences,

Raebareli, India

*Correspondence:

Tianyou Guo

gtyou168@126.com

tThese authors have contributed equally to this work

Specialty section:

This article was submitted to

Public Mental Health,

a section of the journal

Frontiers in Public Health

Received: 16 August 2021 Accepted: 22 October 2021 Published: 25 November 2021

Citation:

Chi X, Huang L, Hall DL, Li R, Liang K, Hossain MM and Guo T (2021) Posttraumatic Stress Symptoms Among Chinese College Students During the COVID-19 Pandemic: A Longitudinal Study.

Front. Public Health 9:759379. doi: 10.3389/fpubh.2021.759379
A longitudinal assessment of the prevalence of posttraumatic stress symptoms (PTSS) and risk factors is indispensable for further prevention and/or treatment. The longitudinal web-based survey enrolled 1,164 college students in China. Measured at two time points (February and August 2020), PTSS, demographic information, adverse childhood experiences (ACEs), resilience and self-compassion information were collected to explicate the prevalence and predictors of PTSS concurrently and over time. Results showed that although PTSS generally declined throughout the 6 months after the outbreak of COVID-19, the prevalence remained relatively high. Resilience and selfcompassion negatively predicted PTSS concurrently and longitudinally. While subjective family socioeconomic status (SES) and ACEs at Wave 1 did not predict PTSS under COVID-19 at Wave 1, but both significantly predicted PTSS at Wave 2. Findings implicate potential targets for detecting and intervening on symptoms of trauma in this vulnerable population.

Keywords: COVID-19, PTSS, longitudinal study, Chinese college students, descriptive survey study

\section{INTRODUCTION}

Posttraumatic stress symptoms (PTSS) are commonly reported after experiencing or witnessing major stressful or distressing events. Its primary symptoms involve flashbacks of related memories, memories, avoidance of reminders of trauma and hypervigilance (1). Notably, in addition to direct exposure to life-threatening events, witnessing traumatic events may also cause PTSS (2). Existed studies of severe infectious respiratory diseases demonstrated that being threatened with infection or witnessing the death or serious injuries of others may conduce to PTSS (3-5). Indeed, the catastrophic consequences (e.g., a surge of critically ill patients, and the paralysis of medical systems) generated by the outbreak of COVID-19 were frequently reported by media (6), which were widely accessed by college students through the mass media, generating vicarious traumatization (7). In addition, due to the infectivity and high fatality rates of COVID-19, governments imposed shut-down measures. Students were confined to home with uncertainties and fear toward the future, which may result in mental health problems, including PTSS (8). Different from most studies on PTSS caused by one-off events, COVID-19-related PTSS is a global 
major public health event, which is found to be mutagenic and highly infectious, causing recurrent outbreaks which still affect people's lives. Furthermore, social media coverage of malignant consequences, such as sudden medical collapse, increases people's awareness of risk and may become chronic stressors.

Frequent and intense PTSS is a core criterion for the diagnosis of posttraumatic stress disorder (PTSD) (9). Furthermore, continually experiencing these symptoms results in weaker social functioning, lower quality of life, and a higher risk for selfharm and suicide (10). After 6 months of the SARS epidemic, the prevalence of PTSS was roughly 32\% among the uninfected Chinese population and $55.1 \%$ among SARS patients (11). Likewise, the coronavirus disease 2019 (COVID-19), has affected many countries as a rapidly spreading infectious disease (12), inflicting substantial mental health problems (13). Studies on PTSS during COVID-19 have indicated that $23-37 \%$ of the general public worldwide are experiencing symptoms of PTSS $(14,15)$. A nationwide survey in China with more than 50 thousand participants found that the prevalence of COVIDrelated PTSS was 35\% (16). Given the fact that COVID-19 may have long-lasting negative effects (17), longitudinal tracking of PTSS is essential as results can help policy-makers implement timely interventions for preventing high-risk behaviors and associated health outcomes.

To mitigate the psychological impacts of the COVID-19 pandemic, it is critical to clarify both the risk factors and protective factors associated with developing PTSS. According to the dynamic stress-vulnerability model (18), many factors can be identified as potential risk factors for the development and persistence of psychopathology (e.g., PTSS). The stressvulnerability model is also known as the diathesis-stress model. The diathesis-stress model was firstly proposed by Meehl (19) to interpret the incidence of schizophrenia. After that, this model was widely used as to investigate other mental health problems $(20,21)$. As research has progressed, diathesis-stress factors have been broadened. No longer limited to biological factors only, multiple factors (e.g., psychological, biological, familial, and social) have also been included. It has prompted a more extensive and systematic examination of the determinants of specific psychiatric disorders. Likewise, the development of PTSS is not due to a single factor, but the result of a combination of multiple factors (22). Therefore, this study attempts to investigate the factors influencing the occurrences and changes of PTSS symptoms in college students using the dynamic stress-vulnerability model as the theoretical framework. These determinants mainly include demographic factors (e.g., age and gender), social vulnerability factors (e.g., socioeconomic status, family structure), and psychobiological vulnerability factors (e.g., adverse childhood experiences, high neuroticism, low resilience and self-compassion). These mentioned variables can be utilized as PTSS predictors among Chinese college students. Females may be more at risk than males for developing PTSS after traumatic events (23). With regard to age, one study found that older adults were more likely to develop post-earthquake PTSS (24).

As for social vulnerability, individuals from non-intact families were observed to be at higher risk for developing psychiatric disorders compared to those from intact families (25).
A recent meta-analysis ( $n=26,715$ participants) examining the relationship between the family socioeconomic status (SES) and psychopathology found that individuals with low SES were more vulnerable to developing mental health problems (26). Chinese youths in rural areas also showed a higher prevalence of PTSS compared to their urban counterparts (27) which are the opposite of a study in Ireland during COVID-19 (3). However, research on the relationships between socio-demographic factors and PTSS under COVID-19 concurrently and over time remained limited.

Regarding psychological vulnerability, adverse childhood experiences (ACEs), as previous studies have shown, are positively correlated with higher PTSS (28). Individuals with childhood adversities are expected to be at greater risk for developing PTSS under COVID-19. However, longitudinal studies of ACEs on PTSS during the pandemic are limited and require further investigation.

Notably, self-compassion has been recognized as one of the most important positive factors in recent years. Self-compassion is the capacity to deliver love, kindness, and caring inward, particularly in distress $(29,30)$. Self-compassion involves being mindfully aware and kind toward oneself and viewing suffering as a larger part of the human experience following exposures to difficulties and hardships (31). High self-compassion is related to less maladaptive coping strategies (32), and presumably an emotion regulation strategy to cope with mental health problems (33). Individuals with high self-compassion tend to have fewer PTSS symptoms (34). Individuals with low self-compassion may be unable to down-regulate the brain's automatic survival response to the fight or flight response after major stressful events (35), resulting in psychological vulnerabilities. According to emotional processing theory (36), trauma information is often not processed appropriately due to avoidance of distress (e.g., triggering fear, which leads to emotional over-arousal). Notably, the level of self-compassion may be an important factor contributing to the development of the PTSS (34). Importantly, self-compassion promotes kind, mindful acceptance of negative emotions, which may be constructive to the gentle exposure of traumatic events as a way to reduce the corresponding PTSS symptoms. This portrays how establishing and maintaining a compassionate perspective may be conducive to developing healthy emotion regulation strategies that help alleviate the negative impacts of trauma.

In addition to self-compassion, resilience is another modifiable factor that attracted researchers' interest. Previous study has observed that individuals with low resilience demonstrated more psychological vulnerabilities to developing PTSS after major negative events (37). In comparison to selfcompassion, the definition of resilience is more complex (38). One of the widely accepted definitions of resilience is the capacity to have hardiness, flexibility, and self-efficacy to adapt under stress (39-41). Individuals with high resilience may have an optimistic attitude toward adversities, helping them cope with negative events and reducing the risk of mental health issues (40). In contrast, low psychological resilience can contribute to more distress (42). Regarding potential psychological factors that may have an influence on PTSS, the emotional processing theory of PTSD suggested self-compassion may alleviate the 
negative influence of COVID-19 on PTSS. Furthermore, a considerable amount of studies showed the importance of resilience on the progression of PTSS $(43,44)$. Clinically, self-compassion and resilience are two important psychological variables which may serve as a way for the intervention of PTSS $(45,46)$. However, research on the longitudinal association between self-compassion, resilience, and COVID-19-related PTSS remains limited. Therefore, this study aims to examine which factors influence PTSS among college students in the context of the COVID-19 pandemic. In addition, to investigate the effect of psychological factors (in the present study resilience and self-compassion) on PTSS symptoms after controlling for demographic and familial factors.

Although previous studies of PTSS in China during stressful events including the COVID-19 outbreak provided valuable information, there are distinct research gaps that require scientific inquiries. Most of the available studies adopt a crosssectional design, which cannot show the change in PTSS after stressful events such as the COVID-19 outbreak. Current longitudinal studies in China only cover a relatively short time frame (e.g., 1 or 2 months) $(47,48)$. Understanding the prevalence of PTSS during COVID-19 and its subsequent change longitudinally with a longer time period may promote more suitable approaches to address these problems. Most studies also focus on the mental health of the general public rather than PTSS in a specific population (e.g., college students). College is an important transition period from late adolescence to adulthood. During this period, young people are prone to encountering psychological crises that can involve feelings of insecurity, suspicion, and disappointment in life, sometimes referred to as a quarter life crisis (49). Thus, it is important to follow the mental health status of this group in a stressful situation such as COVID-19.

Thereby, based on the dynamic stress-vulnerability model and emotional processing theory, two waves of longitudinal data were collected over 6 months (February to August 2020) to develop the following study that has three main goals. The first goal is examining the incidence and variations of COVID-19-related PTSS among college students in China. The second is further investigating whether socio-demographic circumstances predict Chinese college students' PTSS under COVID-19 concurrently and over time. The third goal is to explore whether psychological variables (in this study, ACEs, self-compassion and resilience) are correlated with PTSS among Chinese college students concurrently and longitudinally.

\section{METHODS}

\section{Participants}

Data for the present study was collected mid to late February in 2020 (Wave 1), $\sim 1$ month after the COVID-19 pandemic outbreak. After 6 months, the same subjects were called for the next round of survey in late August, 2020 (Wave 2). Participants were recruited from more than 100 colleges/universities across the country. All students who agreed to take part signed a consent form before filling out the questionnaires. In Wave 1, 1,218 students agreed to participate in the survey and
TABLE 1 | Comparisons of psychosocial variables and PTSS of the participants measured at Wave 1 by loss to follow-up.

\begin{tabular}{|c|c|c|c|c|c|}
\hline \multirow[t]{2}{*}{ Variables } & \multicolumn{2}{|c|}{ Follow-up } & \multicolumn{2}{|c|}{ Loss to follow-up } & \multirow[t]{2}{*}{$p$} \\
\hline & N/M & $\% / S D$ & N/M & $\% / S D$ & \\
\hline Age & 20.48 & 1.80 & 20.64 & 1.99 & 0.17 \\
\hline Gender & & & & & 0.82 \\
\hline Male & 351 & 35.40 & 59 & 34.30 & \\
\hline Female & 641 & 64.60 & 113 & 65.70 & \\
\hline Siblings & & & & & 0.42 \\
\hline One child & 317 & 32.00 & 60 & 34.90 & \\
\hline Non-one child & 675 & 68.00 & 112 & 65.10 & \\
\hline Family intactness & & & & & 0.56 \\
\hline Intactness & 909 & 91.60 & 160 & 93.00 & \\
\hline Non-intactness & 83 & 8.40 & 12 & 7.00 & \\
\hline SES & 4.86 & 1.36 & 4.84 & 1.37 & 0.90 \\
\hline \multicolumn{6}{|l|}{ Residence } \\
\hline Urban & 557 & 56.10 & 90 & 52.32 & 0.59 \\
\hline Rural & 435 & 43.90 & 82 & 47.68 & \\
\hline Resilience & 35.19 & 6.20 & 36.41 & 5.71 & 0.44 \\
\hline Self-compassion & 3.24 & 0.47 & 3.26 & 0.48 & 0.45 \\
\hline ACEs & 1.33 & 1.79 & 1.27 & 1.64 & 0.48 \\
\hline
\end{tabular}

p obtained from Chi-square tests and t-tests.

1,164 students eventually completed the questionnaire. The sample consisted of $410(35.22 \%)$ males and $754(64.78 \%)$ females (mean age $=20.56$ years; $S D=1.90$ ). From Wave 1 to Wave 2, 992 participants stayed in the study out of the original sample size. These participants were verified as the same participants from Wave 1 by matching their phone numbers with our original records. Further demographic information about the participants is summarized in Table 1.

\section{Procedure}

We recruited participants through an online survey. The invited students were asked to complete a questionnaire that included socio-demographic information and scales for subjective SES, self-compassion, adult attachment, and resiliency. After completing the questionnaire, participants received a compensation of $\sim 10 \mathrm{RMB}(\sim 1.5$ USD) which was provided online. There were no missing values due to the setup of the electronic questionnaire that required participants to answer each question. The project obtained ethical clearance from the Human Research Ethics Committee of the first author's affiliation.

\section{Instruments}

\section{Sociodemographic Characteristics}

Participants were invited to report their age, gender $(0=$ male and $1=$ female), family type (intact family or non-intact family), type of residence (urban areas $=0$, rural areas $=1$ ), and sibling status $($ no $=0$, yes $=1)$. 


\section{Subjective Socioeconomic Status}

The widely-used subjective SES scale was adopted to assess participants' subjective socioeconomic status (50). Participants were given a drawing of a ladder with 10 rungs and asked to choose a number that best represented their family's socioeconomic status.

\section{PTSS}

We revised the abbreviated PTSD Checklist-Civilian Version (PCL-C) to assess COVID-19-related PTSS. Item example: "Repeated, disturbing memories, thoughts, or images of COVID19 from the past?" There are 6 items with 5 response options (from $1=$ not at all to $5=$ extremely). Scores range from 6 to 30 , with higher scores indicating higher PTSS. As previous research studies characterized a score of at least 14 as an indication of PTSD (sensitivity 92\%, specificity of 72\%) (22). The Cronbach's $\alpha$ coefficient for the scale in this study was 0.81 in Wave 1 and 0.86 in Wave 2 , respectively.

\section{Self-Compassion}

Participants were given the 26-item self-compassion scale (51) which assesses three aspects of self-compassion (negative aspects are reverse coded), including self-kindness, common humanity and mindfulness. Responses are identified on a 5-point scale from "Almost Never" to "Almost Always." Higher scores indicate higher level of self-compassion. The questionnaire was validated in China previously (52). The Cronbach's $\alpha$ coefficient for the scale in this study was 0.87 .

\section{Resilience}

The Abridged Connor-Davidson Resilience Scale is a selfadministered questionnaire with a single dimension (53). It has 10 items with 5 response options (from $0=$ never to $4=$ almost always). The scale reflects the ability to tolerate stress and adversities. The final score is the sum of the responses for each item (range 0-40) where higher scores indicate higher resiliency. The scale's reliability was validated previously (54) and the Cronbach's $\alpha$ coefficient of the scale in this study was 0.92 .

\section{Adverse Childhood Experiences}

Childhood trauma was assessed by the Chinese version of the Revised Adverse Childhood Experiences Scale (55). There were 14 items in the scale and were dichotomously coded (1 for presence and 0 for absence). Higher scores presented severer childhood adversities.

\section{Statistical Analyses}

First, in order to check whether the loss of subjects had an impact on our results, we compared the data of the lost and retained subjects. Next, the percentages of participants who had PTSS based on screening procedures in the two waves were compared using related-samples $\mathrm{McNemar}$ tests to investigate the change in student PTSS after 6-months. Finally, the first hierarchical regression analysis was carried out to investigate the predictive effects of sociodemographic and psychological factors on PTSS concurrently. Specifically, PTSS was treated as a dependent variable, and demographic variables (i.e., age and gender) were considered independent variables, which were placed in the first
TABLE 2 | Prevalence and change of PTSS in Waves 1 and 2 (February 2020 and August 2020).

\begin{tabular}{lcccc}
\hline $\begin{array}{l}\text { PTS total } \\
\text { score }\end{array}$ & $\begin{array}{c}\text { PTSS } \\
\text { (Cut-off } \geq 14)\end{array}$ & \\
\hline Wave 1 (M/SD) & Wave 2 (M/SD) & Wave 1 (\%) & Wave 2 (\%) & $x^{2}$ \\
$11.79 \pm 4.25$ & $11.38 \pm 4.27$ & 30.80 & 27.40 & $3.35^{\dagger}$ \\
\hline${ }^{+} p=0.06$. & & & \\
\end{tabular}

step. After the controlled for age and gender, familial variables (i.e., family intactness, subjective SES, and residence) were placed in the second step. Thirdly, psychological factors (i.e., ACEs, resilience, and self-compassion) as independent variables were placed in the third step to assess their association with PTSS after controlled for variables in the first and second steps. Likewise, the second hierarchical regression was performed to explore their longitudinal relationships after the control for T1 PTSS in the first step. Statistical analyses were carried out using SPSS 23.0. The statistical significance was set at $p<0.05$ (two-tailed) for the interpretation of the results.

\section{RESULTS}

\section{Comparisons of Followed-Up Participants and Attrition}

$14.78 \%(n=172)$ of the baseline participants did not complete the study in Wave 2 (Table 1). Differences were not statistically significant between the retained and dropped participants. Of the retained participants, the mean age was 20.48 years old $(\mathrm{SD}=$ 1.80). Thirty-five point four percent of participants were male and $68.00 \%$ reported having sibling(s). Approximately $8.40 \%$ of students reported coming from non-intact families and $\sim 56.10 \%$ of participants were from urban areas.

\section{Screening for PTSS}

As can be seen in Table 2, 30.80\% of the college students in Wave 1 were identified as having PTSS while $27.40 \%$ of students in Wave 2 were identified as having PTSS 6 months later. These data suggest that the COVID-19 related PTSS among China's college students is generally high.

\section{Differences of PTSS at Two Time Points}

We performed related-samples McNemar tests to compare PTSS levels at two time points. The results show that the change was borderline significant. Participants showed lower PTSS levels at Wave 2 then at Wave $1\left(x^{2}=3.35, p=0.06\right)$ (Table 2).

\section{Correlates and $T$-Test Results of PTSS in College Students}

In the bivariate correlation analysis, the following variables were correlated with PTSS in Wave 1: age, ACEs, resilience and selfcompassion $(p s<0.05)$. Independent $t$-tests showed significant differences in PTSS between rural and urban areas $(t=2.11, p<$ $0.05)$. There was marginal significance between intact and nonintact families $(t=-1.89, p=0.059)$. In Wave 2, gender, SES, 
TABLE 3 | Correlations or $t$-test results of independent variables with PTSS in Waves 1 and 2 (February 2020 and August 2020).

\begin{tabular}{|c|c|c|c|c|c|c|c|c|c|}
\hline & Age & Gender & Siblings & Family intactness & SES & Residence & ACEs & Resilience & Self-compassion \\
\hline PTSD-Wave 1 & $0.09^{\star \star}$ & -1.75 & 1.26 & $-1.89^{\dagger}$ & 0.01 & $2.11^{*}$ & $0.12^{\star \star \star}$ & $-0.31^{\star \star \star}$ & $-0.32^{\star \star \star}$ \\
\hline PTSD-Wave 2 & 0.00 & $-2.05^{\star}$ & 1.10 & -0.98 & $-0.06^{\star}$ & 0.07 & $0.19^{\star \star \star}$ & $-0.31^{\star \star \star}$ & $-0.31^{\star \star \star}$ \\
\hline
\end{tabular}

${ }^{+} p=0.059$.

${ }^{*} p<0.05 ;{ }^{* *} p<0.01 ;{ }^{* * \star} p<0.001$.

TABLE 4 | Multiple linear regression of predictors of PTSS in Waves 1 and 2 (February 2020 and August 2020).

\begin{tabular}{|c|c|c|c|c|c|c|c|c|c|c|c|}
\hline \multicolumn{6}{|c|}{ Dependent variable: PTSS-Wave 1} & \multicolumn{6}{|c|}{ Dependent variable: PTSS-Wave 2} \\
\hline & & & & & & PTSD-Wave 1 & 0.50 & 0.03 & 0.47 & $16.69^{\star \star \star}$ & \\
\hline Step 1 & & & & & $0.01^{\star \star \star}$ & Step 2 & & & & & 0.00 \\
\hline Demographic factors & & & & & & Demographic factors & & & & & \\
\hline Gender & 0.52 & 0.27 & 0.06 & 1.94 & & Gender & 0.33 & 0.25 & 0.04 & 1.30 & \\
\hline Step 2 & & & & & 0.01 & Step 3 & & & & & $0.01^{*}$ \\
\hline Familial factors & & & & & & Familial factors & & & & & \\
\hline Family intactness & 0.75 & 0.46 & 0.05 & 1.63 & & Family intactness & 0.06 & 0.43 & 0.00 & 0.14 & \\
\hline Subjective SES & -0.01 & 0.10 & 0.00 & -0.06 & & Subjective SES & -0.21 & 0.09 & -0.07 & $-2.32^{*}$ & \\
\hline Resilience & -0.11 & 0.02 & -0.17 & $-4.52^{\star \star \star}$ & & Resilience & -0.08 & 0.02 & -0.12 & $-3.38^{\star \star}$ & \\
\hline Self-compassion & -1.92 & 0.32 & -0.22 & $-5.92^{\star \star \star}$ & & Self-compassion & -0.75 & 0.32 & -0.08 & $-2.32^{*}$ & \\
\hline
\end{tabular}

${ }^{*} p<0.05 ;{ }^{* \star} p<0.01 ;{ }^{* \star *} p<0.001$.

ACEs, resilience and self-compassion were correlated with PTSS $(p s<0.05)$ (Table 3).

\section{Predictors of PTSS in College Students}

The results of hierarchical regression analyses were shown in Table 4. In Wave 1, age positively predicted PTSS as older participants manifested more severe PTSS. Gender could not predict PTSS in both waves $(\beta=0.06, \beta=0.04, p>0.05)$. Residence also positively predicted PTSS as participants in urban areas reported higher PTSS. Resilience and self-compassion significantly predicted PTSD concurrently and longitudinally at 6 months as low amounts of both psychological variables were associated with higher levels of PTSS concurrently $(\beta=-0.17, p$ $<0.001 ; \beta=-0.22, p<0.001)$ and longitudinally $(\beta=-0.12, p$ $<0.001 ; \beta=-0.08, p=0.021)$. Subjective SES did not predict PTSS under COVID-19 in Wave 1. However, it significantly predicted participants' PTSS in Wave $2(\beta=-0.07, p=0.021)$. Likewise, ACEs did not correlate with PTSS in Wave 1 but they significantly predicted PTSS in Wave $2(\beta=0.12, p<0.001)$.

\section{DISCUSSION}

This study showed the prevalence of PTSS under COVID-19 among Chinese college students with an interval of 6 months.
In addition, the concurrent and longitudinal predictive effects of demographic, familial, and psychological factors on PTSS were examined. $30.8 \%$ of Chinese college students reported having PTSS in February but this number went down to $27.4 \%$ in August, 2020. The prevalence of PTSS among college students in China under the COVID-19 pandemic was lower than that after the Wenchuan earthquake among trauma-affected people (45.5\%) (56). However, the prevalence of PTSS was almost as high as that of the SARS outbreak (31.18\%) among the public in epidemic-affected areas (11). This may be because COVID-19 occurred over a longer time period in contrast to the Wenchuan earthquake which was sudden. Individuals are aware that their risk of being infected by COVID-19 can be reduced if the correct precautions are taken, which may lead to a less sense of losing control, resulting in a less severe posttraumatic stress reaction (57). Of note, despite the absence of a large-scale outbreak of COVID-19 in provinces other than Hubei provinces (worsthit area), people in other regions may also be severely affected by the outbreak of COVID-19. The results are in consonance with some existed research on major stress events $(58,59)$. Potential reasons for this include the "amplification" effect of risk events $(58,59)$. Specifically, residents of light and non-affected areas primarily resort to the media to obtain COVID-19-related information. This dissemination through the media or other 
informal pathways may affect an individual's risk perception and thus influences PTSS symptoms.

The prevalence rates of COVID-19-related PTSS in the present study were similar to two studies on the general Italian population (age range: 18-64, and 18-89, respectively), with a prevalence rate of $27.7-35.6 \%(60,61)$. These were close to the prevalence rate of $31.8 \%$ in the third study with American young adults (aged from 18 to 30) during COVID-19 (62). Nevertheless, other research studies on similar samples depict a lower incidence of PTSS. For instance, Karatzias et al. found that $17.7 \%$ of adults (age: 18 to more than 65 ) in Ireland, and $16.79 \%$ in the United Kingdom reported COVID-19-related PTSS (3). One possible explanation of the disparity may be different screening tools adopted in the investigations, with some tools having higher cut-off points. In addition, the severity and development stage of the pandemic locally may also affect PTSS. Future studies can advance the research of PTSS during COVID-19 with representative samples, longitudinal studies, or meta-analysis.

Wave 1 of the study found that age and residence significantly predicted PTSS during COVID-19 in college students. Higher PTSS was reported with increasing age. A possible reason for this may be that older students may be experiencing more stress due to the difficulty of finding employment during the uncertainty of COVID-19 (63). Moreover, the results from the hierarchical regression showed that gender did not predict PTSS in this study, which is inconsistent with previous research (64). This may be due to the fact that, unlike certain traumas, infectious diseases such as COVID-19 are threatening regardless of gender. In addition, type of residence was associated with PTSS in Wave 1 which indicated that college students in urban areas experienced more PTSS compared to their rural counterparts. This may be due to the fact that the outbreak of COVID19 during February, 2020 in China spread more rapidly in major cities with greater population densities. However, age and residence did not predict PTSS in Wave 2. This could be because Wave 2 data was gathered in late August, 2020 when COVID19 was better controlled in China, compared to the situation in February, 2020. Consequently, older students could have more positive outlooks on their employment opportunities and urban residents may no longer fear the rapid spread of the virus in their cities.

Surprisingly, we found that individuals with fewer ACEs and higher SES reported lower levels of PTSS in Wave 2 while the differences were not significant in Wave 1. One possible explanation for this is that at the beginning of the pandemic, all individuals, regardless of their ACEs and SES, may be affected concurrently by the acute stress of the public health emergency. However, as the pandemic progressed and the economy deteriorated, those with higher family affluence have more resources to buffer stress from the economic downturn compared to individuals with low SES (65). Individuals with more ACEs may also have less effective strategies for emotion regulation, making them more vulnerable to negative adjustment longitudinally (66). As a result, despite COVID-19 infection numbers decreasing drastically by Wave 2 in August, 2020, the economic and psychological effects from the virus are long-lasting and likely still deeply impact individuals with higher ACEs and low SES.

We further found that self-compassion was significantly associated with PTSS concurrently and longitudinally as lower levels of self-compassion predicted higher levels of PTSS. These findings are similar to several studies conducted in America $(67,68)$. Self-compassion emphasizes kindness toward one's self and mindful awareness of distressing experiences which activates the mammalian caring system (69). This neurocognitive mechanism may lead to fewer posttraumatic stress symptoms. In addition, individuals with high selfcompassion may be better at self-regulating their stress levels and adapting coping strategies such as constructively reframing or accepting difficulties (70), resulting in less severe PTSS. These findings suggest that individuals with low self-compassion are more psychologically vulnerable and have more risk factors, putting them at greater risk for being psychologically affected by the COVID-19 pandemic.

We also found that greater resiliency negatively predicted PTSS which is in line with previous study (71). One possible reason is that individuals with high resiliency are more adaptive when facing difficulties, enabling them to recover from negative events (72). High levels of resilience can help individuals suffering from trauma recover more quickly (73). As previous research has found, resilience can lead to positive emotions (74) as it can enhance happiness and promote psychological health which buffers negative psychological effects caused by major stressful events. Our results suggest that elevating levels of resiliency can diminish PTSS.

Several limitations should be noted in this study. First, our participants were mainly from Guangdong, Anhui, Hebei, and Jiangsu provinces in China. Survey data from the strongly-affected province (Hubei) accounted for a very small proportion (1.57\%); thus, the findings may not be generalizable across the Hubei province. Furthermore, the present study assessed COVID-related PTSS. Therefore, generalizing the results to PTSS to other major stressors demands caution. Given this study mainly focused on the concurrent and longitudinal association between several demographic, social, and psychological variables on PTSS under the COVID-19 pandemic, the interactions between variables on PTSS were not examined. Future studies can explore their relationships and the underlying mechanism. Moreover, although the Abbreviated PCL checklist showed good psychometric properties in previous studies, the pandemic caused widespread suffering, and the reports from mass media exacerbated vicarious trauma. Given that we did not assess the fear and threat severity of COVID19 among participants, the results may not preclude the possibility of false positives. It is preferable to assess the stress intensity of COVID-19 for each individual in future studies to improve data accuracy. Lastly, the general methodological limitations of self-reported surveys should be considered, which may affect the interpretations of the measured constructs as well as the generalizability of the study's findings. Further studies should be conducted using different methods of data collection to collect objective data (e.g., clinician-rated or bioindicators), minimize methodological biases, and explore 
potential psycho-pathological mechanisms that may complement such advanced investigations.

Despite these limitations, this study has several results in aiding our understanding of PTSS among college students during the COVID-19 pandemic. The study can likely be generalized to most Chinese college students as it is based on a relatively large sample size. The incidence of PTSS among college students in China is relatively high, suggesting that policy-makers, educators, and clinical professionals need to take timely and effective measures to reduce the PTSS of college students thus promoting their healthy development. We found that individuals with low SES and ACEs longitudinally were more prone to develop PTSS under COVID-19, which suggests an immediate need for mental health interventions for this vulnerable population. Additionally, factors such as resilience and self-compassion may be protective factors against the negative effects of stressful events on mental health of college students at a single time point and over time. Recently, there have been calls for synchronous and asynchronous remote delivery of resiliency interventions to address COVID-19 stress (75). Our findings suggest that such programs can be particularly helpful if targeted to college students with histories of trauma or low SES backgrounds. Therefore, prospective measures for improving resiliency and self-compassion in college students may be a way to mitigate the impact of the COVID-19 pandemic on mental health and a strategy to improve general well-being in the future.

\section{REFERENCES}

1. Deja M, Denke C, Weber-Carstens S, Schröder J, Pille CE, Hokema F, et al. Social support during intensive care unit stay might improve mental impairment and consequently health-related quality of life in survivors of severe acute respiratory distress syndrome. Crit Care. (2006) 10:R147. doi: 10.1186/cc5070

2. Patki G, Salvi A, Liu H, Salim S. Witnessing traumatic events and posttraumatic stress disorder: Insights from an animal model. Neurosci Lett. (2015) 600:28-32. doi: 10.1016/j.neulet.2015.05.060

3. Karatzias T, Shevlin M, Murphy J, Mcbride O, Ben Ezra M, Bentall RP, et al. Posttraumatic stress symptoms and associated comorbidity during the COVID-19 pandemic in Ireland: a population-based study. J Trauma Stress. (2020) 33:365-70. doi: 10.1002/jts.22565

4. Hong S, Kim H, Park MK. Impact of COVID-19 on post-traumatic stress symptoms in the general population: an integrative review. Int J Ment Health Nurs. (2021) 30:834-46. doi: 10.1111/inm.12875

5. Wu P, Fang Y, Guan Z, Fan B, Kong J, Yao Z, et al. The psychological impact of the SARS epidemic on hospital employees in China: exposure, risk perception, and altruistic acceptance of risk. Can J Psychiatry. (2009) 54:302-11. doi: 10.1177/070674370905400504

6. Kadam AB, Atre SR. Negative impact of social media panic during the COVID-19 outbreak in India. J Travel Med. (2020) 27:a57. doi: 10.1093/jtm/taaa057

7. Liu C, Liu Y. Media exposure and anxiety during COVID-19: the Mediation Effect of Media Vicarious Traumatization. Int J Environ Res Public Health. (2020) 17:4720. doi: 10.3390/ijerph17134720

8. Tang W, Hu T, Hu B, Jin C, Wang G, Xie C, et al. Prevalence and correlates of PTSD and depressive symptoms one month after the outbreak of the COVID19 epidemic in a sample of home-quarantined Chinese university students. $J$ Affect Disorders. (2020) 274:1-7. doi: 10.1016/j.jad.2020.05.009

9. Biggs QM, Ursano RJ, Wang J, Krantz DS, Carr RB, Wynn GH, et al. Daily variation in post-traumatic stress symptoms in individuals with and

\section{DATA AVAILABILITY STATEMENT}

The raw data supporting the conclusions of this article will be made available by the authors, without undue reservation.

\section{ETHICS STATEMENT}

The studies involving human participants were reviewed and approved by the project obtained ethical clearance from the Human Research Ethics Committee of the Shenzhen University (No: 2020005). The patients/participants provided their written informed consent to participate in this study.

\section{AUTHOR CONTRIBUTIONS}

$\mathrm{XC}, \mathrm{LH}$, and TG took a principal role in designing the study, writing the protocol, developing methodologies, and drafting the manuscript. LH performed the statistical analysis. DH, RL, $\mathrm{KL}$, and $\mathrm{MH}$ edited the manuscript. All authors have read and approved the final manuscript.

\section{FUNDING}

TG was supported by National Natural Science Foundation of China (31871115). XC was supported by Guangdong Basic and Applied Basic Research Foundation (2021A1515011330).

without probable post-traumatic stress disorder. Bmc Psychiatry. (2019) 19:18. doi: 10.1186/s12888-019-2041-7

10. Liang L, Ren $\mathrm{H}$, Cao $\mathrm{R}, \mathrm{Hu} \mathrm{Y}$, Qin Z, Li C, et al. The effect of COVID-19 on youth mental health. Psychiat Quart. (2020) 91:84152. doi: 10.1007/s11126-020-09744-3

11. Xu Y, Zhang KR, Liu ZG, Yang H, Song LP, Xue YZ. A controlled study of PTSD in SARS patients and the public in infected areas. Chin J Clin Psychol. (2005) 13:210-2. doi: 10.3201/eid1007.030703

12. World Health Organization. Statement on the Second Meeting of the International Health Regulations Emergency Committee regarding the Outbreak of Novel Coronavirus (2019-nCoV) (2020). Available online at: https://www.who.int/news-room/detail/30-01-2020-statement-onthe-second-meeting-of-the-international-health-regulations-(2005)emergency-committee-regarding-the-outbreak-of-novel-coronavirus(2019-ncov) (accessed July 20, 2021).

13. Wu T, Jia X, Shi H, Niu J, Yin X, Xie J, et al. Prevalence of mental health problems during the COVID-19 pandemic: a systematic review and metaanalysis. J Affect Disord. (2020) 281:91-8. doi: 10.1016/j.jad.2020.11.117

14. Alshehri FS, Alatawi Y, Alghamdi BS, Alhifany AA, Alharbi A. Prevalence of post-traumatic stress disorder during the COVID-19 pandemic in Saudi Arabia. Saudi Pharm J. (2020) 28:1666-73. doi: 10.1016/j.jsps.2020. 10.013

15. Rossi R, Socci V, Talevi D, Mensi S, Niolu C, Pacitti F, et al. COVID19 Pandemic and Lockdown Measures Impact on Mental Health Among the General Population in Italy. Front Psychiatry. (2020) 11:790. doi: 10.3389/fpsyt.2020.00790

16. Qiu J, Shen B, Zhao M, Wang Z, Xu Y. A nationwide survey of psychological distress among Chinese people in the COVID-19 epidemic: implications and policy recommendations. General Psychiatry. (2020) 33:100213. doi: 10.1136/gpsych-2020-100213

17. Centers for Disease Control and Prevention. Long-Term Effects of COVID19. (2020). Available online at: https://www.cdc.gov/coronavirus/2019-ncov/ long-term-effects.html (accessed June 20, 2021). 
18. Spijker J, de Graaf R, Bijl RV, Beekman ATF, Ormel J, Nolen WA. Determinants of persistence of major depressive episodes in the general population. Results from the Netherlands Mental Health Survey and Incidence Study (NEMESIS). J Affect Disorders. (2004) 81:231-40. doi: 10.1016/j.jad.2003.08.005

19. Meehl PE. Schizotaxia, schizotypy, schizophrenia. Am Psychol. (1962) 17:827-38. doi: 10.1037/H0041029

20. Gelaye B, Kajeepeta S, Williams MA. Suicidal ideation in pregnancy: an epidemiologic review. Arch Womens Ment Health. (2016) 19:74151. doi: 10.1007/s00737-016-0646-0

21. Schotte CKW, Van Den Bossche B, De Doncker D, Claes S, Cosyns P. A biopsychosocial model as a guide for psychoeducation and treatment of depression. Depress Anxiety. (2006) 23:312-24. doi: 10.1002/da.20177

22. Ni MY, Yao XI, Leung KSM, Yau C, Leung CMC, Lun P, et al. Depression and post-traumatic stress during major social unrest in Hong Kong: a 10-year prospective cohort study. Lancet. (2020) 395:27384. doi: 10.1016/S0140-6736(19)33160-5

23. Olff M. Sex and gender differences in post-traumatic stress disorder: an update. Eur $J$ Psychotraumatol. (2017) 8(sup. 4):1351204. doi: 10.1080/20008198.2017.1351204

24. Zhang N, Zhang Y, Wu K, Chen Z, Liu Y, Zhu Z. Post-traumatic stress disorder and correlates in survivors of Wenchuan earthquake. Chin J Clin Psychol. (2010) 13:23-9. doi: 10.16128/j.cnki.1005-3611.2010.01.012

25. D'Onofrio B, Emery R. Parental divorce or separation and children's mental health. World Psychiatry. (2019) 18:100. doi: 10.1002/wps.20590

26. Peverill M, Dirks MA, Narvaja T, Herts KL, Comer JS, McLaughlin KA. Socioeconomic status and child psychopathology in the United States: a meta-analysis of population-based studies. Clin Psychol Rev. (2021) 83:101933. doi: 10.1016/j.cpr.2020.101933

27. Hou T, Mao X, Dong W, Cai W, Deng G. Prevalence of and factors associated with mental health problems and suicidality among senior high school students in rural China during the COVID-19 outbreak. Asian J Psychiatr. (2020) 54:102305. doi: 10.1016/j.ajp.2020.102305

28. Messman Moore TL, Bhuptani PH. A review of the long-term impact of child maltreatment on posttraumatic stress disorder and its comorbidities: an emotion dysregulation perspective. Clin Psychol:Sci Pract. (2017) 24:15469. doi: $10.1111 /$ cpsp. 12193

29. Neff KD, Long P, Knox MC, Davidson O, Kuchar A, Costigan A, et al. The forest and the trees: examining the association of self-compassion and its positive and negative components with psychological functioning. Self Identity. (2018) 6:627-45. doi: 10.1080/15298868.2018.1436587

30. Neff KD, Dahm KA. Self-compassion: what it is, what it does, and how it relates to mindfulness. In: Ostafin BD, Robinson MD, Meier BP, editors. Handbook of Mindfulness and Self-Regulation. New York, NY: Springer (2015). p. 121-37. doi: 10.1007/978-1-4939-2263-5_10

31. Bluth K, Neff KD. New frontiers in understanding the benefits of self-compassion. Self Identity. (2018) 17:6058. doi: 10.1080/15298868.2018.1508494

32. Ewert C, Vater A, Schröder-Abé M. Self-Compassion and Coping: a metaanalysis. Mindfulness. (2021) 12:1063-77. doi: 10.1007/s12671-020-01563-8

33. Diedrich A, Grant M, Hofmann SG, Hiller W, Berking M. Self-compassion as an emotion regulation strategy in major depressive disorder. Behav Res Ther. (2014) 58:43-51. doi: 10.1016/j.brat.2014.05.006

34. Winders SJ, Murphy O, Looney K, O'Reilly G. Self-compassion, trauma, and posttraumatic stress disorder: a systematic review. Clin Psychol Psychother. (2020) 27:300-29. doi: 10.1002/cpp.2429

35. Gilbert P. The origins and nature of compassion focused therapy. Br J Clin Psychol. (2014) 53:6-41. doi: 10.1111/bjc.12043

36. Foa EB, Steketee G, Rothbaum BO. Behavioral/cognitive conceptualizations of post-traumatic stress disorder. Behav Ther. (1989) 20:155-76. doi: 10.1016/S0005-7894(89)80067-X

37. Yuan G, Xu W, Liu Z, An Y. Resilience, posttraumatic stress symptoms, and posttraumatic growth in Chinese adolescents after a tornado: the role of mediation through perceived social support. J Nerv Ment Dis. (2018) 206:130-5. doi: 10.1097/NMD.0000000000000778

38. Southwick SM, Bonanno GA, Masten AS, Panter-Brick C, Yehuda R. Resilience definitions, theory, and challenges: interdisciplinary perspectives. Eur J Psychotraumatol. (2014) 5:25338. doi: 10.3402/ejpt.v5.25338
39. Smith BW, Dalen J, Wiggins K, Tooley E, Christopher P, Bernard J. The brief resilience scale: assessing the ability to bounce back. Int J Behav Med. (2008) 15:194-200. doi: 10.1080/10705500802222972

40. Connor KM, Davidson JRT. Development of a new resilience scale: the Connor-Davidson Resilience Scale (CD-RISC). Depress Anxiety. (2003) 18:7682. doi: 10.1002/da.10113

41. Werner EE. Risk, resilience, and recovery: perspectives from the Kauai Longitudinal Study. Dev Psychopathol. (1993) 5:50315. doi: $10.1017 / S 095457940000612 \mathrm{X}$

42. Xi Y, Yu H, Yao Y, Peng K, Wang Y, Chen R. Post-traumatic stress disorder and the role of resilience, social support, anxiety and depression after the Jiuzhaigou earthquake: a structural equation model. Asian J Psychiatr. (2020) 49:101958. doi: 10.1016/j.ajp.2020.101958

43. Galatzer-Levy IR, Huang SH, Bonanno GA. Trajectories of resilience and dysfunction following potential trauma: a review and statistical evaluation. Clin Psychol Rev. (2018) 63:41-55. doi: 10.1016/j.cpr.2018.05.008

44. Agaibi CE, Wilson JP. Trauma, PTSD, and resilience. Trauma Violence Abuse. (2005) 6:195-216. doi: 10.1177/1524838005277438

45. Wrenn GL, Wingo AP, Moore R, Pelletier T, Gutman AR, Bradley B, et al. The effect of resilience on posttraumatic stress disorder in traumaexposed inner-city primary care patients. J Natl Med Assoc. (2011) 103:5606. doi: 10.1016/S0027-9684(15)30381-3

46. Luo X, Che X, Lei Y, Li H. Investigating the influence of self-compassionfocused interventions on posttraumatic stress: a systematic review and metaanalysis. Mindfulness. (2021) 9:1816-24. doi: 10.1007/s12671-021-01732-3

47. Duan H, Yan L, Ding X, Gan Y, Kohn N, Wu J. Impact of the COVID-19 pandemic on mental health in the general Chinese population: changes, predictors and psychosocial correlates. Psychiat Res. (2020) 293:113396. doi: 10.1016/j.psychres.2020.113396

48. Wang C, Pan R, Wan X, Tan Y, Xu L, McIntyre RS, et al. A longitudinal study on the mental health of general population during the COVID-19 epidemic in China. Brain Behav Immun. (2020) 87:40-8. doi: 10.1016/j.bbi.2020.04.028

49. Robinson OC. A longitudinal mixed-methods case study of quarter-life crisis during the post-university transition: Lockedout and locked-in forms in combination. Emerg Adulthood. (2019) 7:167-79. doi: 10.1177/2167696818764144

50. Ostrove JM, Adler NE, Kuppermann M, Washington AE. Objective and subjective assessments of socioeconomic status and their relationship to selfrated health in an ethnically diverse sample of pregnant women. Health Psychol. (2000) 19:613. doi: 10.1037/0278-6133.19.6.613

51. Neff KD. The development and validation of a scale to measure selfcompassion. Self Identity. (2003) 2:223-50. doi: 10.1080/15298860309027

52. Chen J, Yan L, Zhou L. Reliability and validity of Chinese version of self-compassion scale. Chin J Clin Psychol. (2011) 19:110-2. doi: 10.16128/j.cnki.1005-3611.2011.06.006

53. Campbell-Sills L, Stein MB. Psychometric analysis and refinement of the Connor-Davidson Resilience Scale (CD-RISC): validation of a 10-item measure of resilience. J Trauma Stress. (2010) 20:1019-28. doi: 10.1002/jts.20271

54. Ye ZJ, Ruan XL, Zeng Z, Xie Q, Cheng MH, Peng CH, et al. Reliability and validity of Chinese version 10 item psychological resilience scale in nursing students. J Nurs. (2016) 23:9-13. doi: 10.16460/j.issn1008-9969.2016.21.009

55. Wang Y, Lin P, Cao F. Validity and reliability of the Chinese version of the revised adverse childhood experience questionnaire. (2018) 32:760-4. doi: 10.3969/j.issn.1000-6729.2018.09.010

56. Kun P, Chen X, Han S, Gong X, Chen M, Zhang W, et al. Prevalence of posttraumatic stress disorder in Sichuan Province, China after the 2008 Wenchuan earthquake. Public Health. (2009) 123:703-7. doi: 10.1016/j.puhe.2009. 09.017

57. Gershuny BS, Cloitre M, Otto MW. Peritraumatic dissociation and PTSD severity: do event-related fears about death and control mediate their relation? Behav Res Ther. (2003) 41:157-66. doi: 10.1016/S0005-7967(01)00134-6

58. Maderthaner R, Guttmann G, Swaton E, Otway HJ. Effect of distance upon risk perception. J Appl Psychol. (1978) 63:380-2. doi: 10.1037/0021-9010.63.3.380

59. Li S, Rao L, Ren X, Bai X, Zheng R, Li J, et al. Psychological typhoon eye in the 2008 Wenchuan earthquake. PLoS ONE. (2009) 4:e9727. doi: 10.1371/JOURNAL.PONE.0004964 
60. Di Crosta A, Palumbo R, Marchetti D, Ceccato I, La Malva P, Maiella R, et al. Individual differences, economic stability, and fear of contagion as risk factors for PTSD symptoms in the COVID-19 emergency. Front Psychol. (2020) 11:567367. doi: 10.3389/fpsyg.2020.567367

61. Forte G, Favieri F, Tambelli R, Casagrande M. The enemy which sealed the world: effects of COVID-19 diffusion on the psychological state of the Italian Population. J Clin Med. (2020) 9:1802. doi: 10.3390/jcm9061802

62. Liu CH, Zhang E, Wong GTF, Hyun S, Hahm HC. Factors associated with depression, anxiety, and PTSD symptomatology during the COVID-19 pandemic: clinical implications for U.S. young adult mental health. Psychiat Res. (2020) 290:113172. doi: 10.1016/j.psychres.2020.113172

63. Li T, Sun Y, Wu Z, Shan N. An empirical study on the employment situation of Chinese college graduates under the impact of COVID-19. J East China Norm Univ:Educ Sci Ed. (2020) 38:110. doi: 10.16382/j.cnki.1000-5560.2020. 10.010

64. Irish LA, Fischer B, Fallon W, Spoonster E, Sledjeski EM, Delahanty DL. Gender differences in PTSD symptoms: an exploration of peritraumatic mechanisms. J Anxiety Disord. (2011) 25:209-16. doi: 10.1016/j.janxdis.2010.09.004

65. Santos RMD. Isolation, social stress, low socioeconomic status and its relationship to immune response in Covid-19 pandemic context. Brain Behav Immun Health. (2020) 7:100103. doi: 10.1016/j.bbih.2020.100103

66. Espeleta HC, Sharkey CM, Bakula DM, Gamwell KL, Mullins LL. Adverse childhood experiences and chronic medical conditions: emotion dysregulation as a mediator of adjustment. J Clin Psychol Med Settings. (2020) 27:572-81. doi: 10.1007/s10880-019-09639-x

67. Hiraoka R, Meyer EC, Kimbrel NA, DeBeer BB, Gulliver SB, Morissette SB. Self-compassion as a prospective predictor of PTSD symptom severity among trauma-exposed US Iraq and Afghanistan war veterans. J Trauma Stress. (2015) 28:127-33. doi: 10.1002/jts.21995

68. Thompson BL, Waltz J. Self-compassion and PTSD symptom severity. J Traum Stress. (2008) 21:556-8. doi: 10.1002/jts.20374

69. Gilbert P. Explorations into the nature and function of compassion. Curr Opin Psychol. (2019) 28:108-14. doi: 10.1016/j.copsyc.2018.12.002

70. Lau BH, Chan CL, Ng S. Self-compassion buffers the adverse mental health impacts of COVID-19-related threats: results from a cross-sectional survey at the first peak of Hong Kong's outbreak. Front Psychiatry. (2020) 11:1203. doi: 10.3389/fpsyt.2020.585270

71. Horn SR, Charney DS, Feder A. Understanding resilience: new approaches for preventing and treating PTSD. Exp Neurol. (2016) 284:119-32. doi: 10.1016/j.expneurol.2016.07.002

72. Garmezy N. Resiliency and vulnerability to adverse developmental outcomes associated with poverty. Am Behav Sci. (1991) 34:41630. doi: 10.1177/0002764291034004003

73. Poole JC, Dobson KS, Pusch D. Childhood adversity and adult depression: the protective role of psychological resilience. Child Abuse Negl. (2017) 64:89100. doi: 10.1016/j.chiabu.2016.12.012

74. Ong AD, Bergeman CS, Bisconti TL, Wallace KA. Psychological resilience, positive emotions, and successful adaptation to stress in later life. J Pers Soc Psychol. (2006) 91:730. doi: 10.1037/0022-3514.91.4.730

75. Hall DL, Millstein RA, Luberto CM, Perez GK, Park ER. Responding to COVID-19 Stress: disseminating mind-body resiliency approaches. Glob Adv Health Med. (2020) 9:804391817. doi: 10.1177/2164956120976554

Conflict of Interest: The authors declare that the research was conducted in the absence of any commercial or financial relationships that could be construed as a potential conflict of interest.

Publisher's Note: All claims expressed in this article are solely those of the authors and do not necessarily represent those of their affiliated organizations, or those of the publisher, the editors and the reviewers. Any product that may be evaluated in this article, or claim that may be made by its manufacturer, is not guaranteed or endorsed by the publisher.

Copyright (C) 2021 Chi, Huang, Hall, Li, Liang, Hossain and Guo. This is an openaccess article distributed under the terms of the Creative Commons Attribution License (CC BY). The use, distribution or reproduction in other forums is permitted, provided the original author(s) and the copyright owner(s) are credited and that the original publication in this journal is cited, in accordance with accepted academic practice. No use, distribution or reproduction is permitted which does not comply with these terms. 